\title{
Evaluation of febrile neutropenic episodes in adult patients with solid tumors
}

\author{
OKTAY YAPICI $^{1}$, FILIZ GUNSEREN ${ }^{1}$, HAFIZE YAPICI ${ }^{1}$, \\ ALPARSLAN MERDIN $^{2}$, ÜLKÜ ÜSER YAYLALI ${ }^{1}$ and FATMA AVCI MERDIN ${ }^{2}$
}

Departments of ${ }^{1}$ Infectiology and Microbiology and ${ }^{2}$ Internal Medicine, Akdeniz University Hospital, Antalya 07059, Turkey

Received October 8, 2015; Accepted December 7, 2015

DOI: $10.3892 / \mathrm{mco} .2015 .722$

\begin{abstract}
The clinical use of cytotoxic chemotherapeutic agents has increased survival in cancer patients. However, treatment-associated bone marrow suppression and neutropenia often render patients prone to life-threatening infections. The aim of this study was to evaluate episodes of febrile neutropenia $(\mathrm{FN})$ in patients with solid tumors, and identify the microorganisms and the factors affecting mortality. A total of 100 primary febrile attacks in cancer patients who were followed up at the Department of Oncology of the Akdeniz University Medical Faculty Hospital between January, 2011 and May, 2012, were retrospectively investigated. FN attacks were classified in three groups as follows: Fever of unknown origin, clinically documented infections and microbiologically documented infections. We found that prolonged neutropenia, Multinational Association for Supportive Care in Cancer (MASCC) score $<21$ and the presence of metastasis increased mortality. We also compared the three groups of infection categories according to mortality rate, but did not observe any significant differences among these groups. Patients with malignancies should be assessed individually during the FN episodes. It is crucial to keep possible infectious pathogens in mind and evaluate the MASCC score, neutropenia duration and metastatic status of the patients, and start empirical antibiotic therapy immediately.
\end{abstract}

\section{Introduction}

Febrile neutropenia (FN) is the most common complication of cytotoxic cancer therapy, with $10-50 \%$ of patients with solid tumors and $>80 \%$ of patients with hematological malignancies developing FN during chemotherapy cycles (1).

The clinical use of cytotoxic chemotherapeutic agents has improved survival in cancer patients. However, treatment-associated bone marrow suppression and neutropenia often render patients prone to life-threatening infections. In patients with

Correspondence to: Dr Oktay Yapici, Department of Infectiology and Microbiology, Akdeniz University Hospital, Dumlupınar Bulvar1, Antalya 07059, Turkey

E-mail: yapicio@hotmail.com

Key words: febrile neutropenia, risc score, solid tumor
FN, infections progress faster and increase mortality (2). After assessing the patients and considering the risk factors, antibiotic treatment should be started immediately.

There is a diversity of risk parameters and scoring systems used in FN patients. The Multinational Association for Supportive Care in Cancer (MASCC), which was developed by Klastersky et al and published in the Journal of Clinical Oncology in 2000, has been widely used for the evaluation of FN patients (3-5).

According to the MASCC scoring system, the patients are divided into low- and high-risk groups, with scores $>21$ considered as low-risk and scores $<21$ as high-risk. Low-risk FN patients have a better recovery without serious complications, and high-risk FN patients develop more complications with a higher mortality rate $(6,7)$. High-risk patients require inpatient treatment with wide-spectrum i.v. antibiotics effective against Pseudomonas aeruginosa ( $P$. aeruginosa) and other serious gram-negative pathogens (8-11).

The aim of this study was to evaluate FN episodes in patients with solid tumors and identify the microorganisms and the factors affecting mortality.

\section{Patients and methods}

Patients. A total of 100 primary FN episodes in 100 cancer patients were retrospectively evaluated. All the patients had been hospitalized at the Department of Oncology, Akdeniz University Hospital (Antalya, Turkey) between January, 2011 and May, 2012. The patients were also followed up at the Department of Infectious Diseases and Clinical Microbiology.

Data collection. All patient data were collected using a FN patient tracking form. The demographic characteristics, primary disease, age, gender, status of the underlying disease, initial absolute neutrophil count, neutropenia duration, neutropenic fever risk classification, infection status and culture results of all the patients were recorded in the forms.

Statistical analysis. Statistical analysis was performed using the SPSS 18.0 statistical software package (SPSS Inc., Chicago, IL, USA).

The distribution characteristics of all the data were analysed as descriptive statistics [mean \pm standard deviation (SD) or median, distribution range and percentage values]. Binary 
Table I. Binary logistic regression analysis of risk factors possibly affecting mortality following a primary episode of febrile neutropenia.

\begin{tabular}{|c|c|c|c|}
\hline Risk factors & Surviving patients, no. & Exitus, no. & P-value \\
\hline Age $>65$ years & 30 & 3 & 0.320 \\
\hline Gender & & & 1 \\
\hline Male & 53 & 5 & \\
\hline Female & 38 & 4 & \\
\hline Underlying primary disease & & & 0.441 \\
\hline Disease status & & & 0.026 \\
\hline Presence of metastases & 57 & 9 & \\
\hline Severity of neutropenia $\left(/ \mathrm{mm}^{3}\right)$ & & & 0.322 \\
\hline $500-1,000$ & 10 & 1 & \\
\hline $100-500$ & 51 & 3 & \\
\hline$<100$ & 30 & 5 & \\
\hline Duration of neutropenia $>5$ days & 7 & 3 & 0.013 \\
\hline MASCC score & & & 0.001 \\
\hline$<21$ & 4 & 8 & \\
\hline$\geq 21$ & 87 & 1 & \\
\hline Infection category & & & 0.073 \\
\hline FUO & 61 & 4 & \\
\hline ICD & 15 & 1 & \\
\hline MDI & 15 & 4 & \\
\hline
\end{tabular}

MASCC, Multinational Association for Supportive Care in Cancer; FUO, fever of unknown origin; ICD, clinically documented infection; MDI, microbiologically documented infection.

logistic regression analysis was used to determine the risk factors for mortality.

Differences between gender were analysed by the Chi-square test. The risk factors for mortality (such as severity and duration of neutropenia, evaluated by the MASCC score) were analyzed by Student's t-test or the Fisher's exact test. P-values $<0.05$ were considered to indicate statistically significant differences.

Patients with an absolute neutrophil count $<500 / \mathrm{mm}^{3}$ and those with an absolute neutrophil count of $500-1,000 / \mathrm{mm}^{3}$ expected to decrease to $<500 / \mathrm{mm}^{3}$ within the following 24-48 $\mathrm{h}$ were included in this study. All the patients had one axillary body temperature measurement $>38.3^{\circ} \mathrm{C}$, or a body temperature between 38.0 and $38.2^{\circ} \mathrm{C}$ for at least $1 \mathrm{~h}$.

The infection categories of $\mathrm{FN}$ patients were stratified into three groups as follows: i) Fever of unknown origin (FUO), defined as neutropenic cases with a fever $>38.3^{\circ} \mathrm{C}$, without any clinically or microbiologically defined infection after 3 days of screening; ii) clinically documented infection (CDI), defined as clinically identified infections (e.g., pneumonia and perianal infection) without detection of any microbiological pathogens; and iii) microbiologically documented infection (MDI), defined as positive blood culture and clinically identified infection supported by culture results (12).

\section{Results}

Patient characteristics. The median age of the patients was 58 years (range, $21-83$ years). Of the 100 patients, 58 were men and 42 were women. The most common primary malignancy of the patients was lung cancer $(n=30)$. Among women, the most common malignancy was breast cancer $(n=18)$. The mortality rate for all our patients was $9 \%$.

The MASCC scoring system was used to determine the risk group in all cases. A total of $12 \%$ of all the patients were classified in the high-risk group (score <21) and $88 \%$ in the low-risk group (score $\geq 21$ ).

When determining mortality, the positive predictive value of the MASCC score was $66.7 \%$ and the negative predictive value was $98 \%$. In addition, the sensitivity of the MASCC score was found to be $88.9 \%$ and the specificity $95.6 \%$.

Of the 100 total FN episodes, $19 \%$ were assessed as MDI, $16 \%$ as CDI and $65 \%$ as FUO.

The most frequent infections were bloodstream infections (11\%), lung infections (9\%) and urinary tract infections (6\%).

Absolute neutrophil counts and duration of neutropenia were also evaluated. Of all the febrile neutropenia episodes, $35 \%$ had absolute neutrophil counts $<100 / \mathrm{mm}^{3}$, $54 \% 100-500 / \mathrm{mm}^{3}$ and $11 \% 500-1,000 / \mathrm{mm}^{3}$. The duration of the neutropenia was found to be $<7$ days in 97 (97\%) of all the FN episodes and $>7$ days in the remaining $3(3 \%)$ cases, with a mean duration of 2.8 days (range, $1-8$ days).

Microbiological analysis. A microbiologically identified focus of infection was detected in 19 of all FN episodes. In the blood and other culture isolates of these patients, 17 of the pathogenic microorganisms were bacterial and 2 were fungal. Of the all 
the isolates, $14(73.6 \%)$ were gram-negative bacterial agents, $3(15.7 \%)$ were gram-positive bacterial agents and $2(10.5 \%)$ were fungal agents. The most commonly isolated gram-negative bacteria were $P$. aeruginosa $(36 \%)$ and Escherichia coli (26\%). The most commonly isolated gram-positive bacteria were methicillin-susceptible Staphylococcus aureus and Enterococcus faecalis. Both fungal isolates were on urinary cultures, and the agents belonged to the Candida species.

Effects of risk factors on mortality. The risk factors for mortality in patients with primary FN episodes were evaluated. We found that prolonged neutropenia, MASCC score $<21$ and the presence of metastasis increased mortality. We also compared the three infection categories according to mortality rate, but we did not observe any significant differences among these three groups. During the total 100 primary FN episodes 9 patients succumbed (Table I). Finally, we did not observe any significant effect of age, gender, underlying primary disease or disease status on mortality.

\section{Discussion}

FN episodes are encountered in a number of cancer types. Many factors affecting the $\mathrm{FN}$ risk have been reported and there is an increasing scientific concern in this area (1-14). The risks of mortality, morbidity and infection may differ between solid organ (e.g., ovary and breast) cancers treated with low-dose chemotherapy without prolonged neutropenia, hematopoietic stem cell transplantation patients and hematological malignancy patients who receive aggressive chemotherapy with neutropenia prolonged for weeks $(7,8)$. Due to this variability, attempts have been made in recent years to determine the patient's risk of infection $(7,8)$.

In our study, prolonged neutropenia and MASCC score $<21$ were found to be significantly associated with mortality $(\mathrm{P}<0.001)$. The severity of the neutropenia did not exert a significant effect on mortality $(\mathrm{P}=0.322)$. Neutropenia duration was not considered as a measure of risk in the MASCC assessment scheme; however, Paesmans (13) reported that neutropenia duration is an important risk factor for mortality. In a study conducted at a cancer center in the USA, it was demonstrated that neutropenia duration $<7$ days decreased mortality rate (14). We compared neutropenia duration $<5$ days with $>5$ days and also found that neutropenia duration $>5$ days was associated with an increased mortality rate.

Günalp et al (15) conducted a study on patients with FN and demonstrated that a MASCC score $<21$ increased mortality $(\mathrm{P}<0.001)$, although they did not observe a significant effect of the severity of neutropenia on mortality $(\mathrm{P}=0.196)(15)$. Their results are similar to ours; however, Günalp et al reported a mortality rate of $32 \%$, whereas in our study the mortality rate was $9 \%$. This difference in mortality rate may be attributed to the hematological malignancy patients included in the study of Günalp et al (15).

Gram-negative pathogens were found to be the dominant pathogens of FN episodes in studies conducted during the 1970s, 80s and 90s; however, gram-positive organisms have become more common due to the increased use of permanent plastic venous catheters, which allow colonization of the gram-positive skin flora and may provide access to the skin flora (16-18). Recently, gram-positive staphylococci have been among the most common bacteremia agents (19). In our study gram-negative bacteria were the most common pathogens, which is likely due to the the low rate of usage of central venous catheters in our patients. Fungi are rare pathogenic agents in neutropenia of shorter duration. We encountered only 3 patients who had a neutropenia duration of $>7$ days, which may explain the low incidence of fungal infections.

Patients with oncological malignancies should be individually assessed during the FN episodes. It is important to keep possible infectious pathogens in mind when evaluating MASCC score, neutropenia duration and metastatic status of the patients, and start empirical antibiotic therapy immediately.

\section{References}

1. Klastersky J: Management of fever in neutropenic patients with different risks of complications. Clin Infect Dis 39 (Suppl 1): S32-S37, 2004.

2. González-Barca E, Fernández-Sevilla A, Carratalá J, Salar A, Peris J, Grañena A and Gudiol F: Prognostic factors influencing mortality in cancer patients with neutropenia and bacteremia. Eur J Clin Microbiol Infect Dis 18: 539-544, 1999.

3. Klastersky J, Paesmans M, Rubenstein EB, Boyer M, Elting L, Feld R, Gallagher J, Herrstedt J, Rapoport B, Rolston K and Talcott J: The Multinational Association for Supportive Care in Cancer risk index: A multinational scoring system for identifying low-risk febrile neutropenic cancer patients. J Clin Oncol 18: 3038-3051, 2000.

4. Paesmans M: Risk factors assesment in febrile neutropenia. Int J Antimicrob Agents 16: 107-111, 2000.

5. Kern WV: Risk assesment and risk-based therapeutic strategies in febrile neutropenia. Curr Opin Infect Dis 14: 415-422, 2001.

6. Ahn S and Lee YS: Predictive factors for poor prognosis febrile neutropenia. Curr Opin Oncol 24: 376-380, 2012.

7. Hui EP, Leung LK, Poon TC, Mo F, Chan VT, Ma AT, Poon A, Hui EK, Mak SS, Lai M, et al: Prediction of outcome in cancer patients with febrile neutropenia: A prospective validation of the multinational association for supportive care in cancer risk index in a Chinese population and comparison with the Talcott model and artificial neural network. Support Care Cancer 19: 1625-1635, 2011.

8. Glasmacher A, von Lilienfeld-Toal M, Schulte S, et al: An evidence based evaluation of important aspects of empirical antibiotic therapy in febrile neutropenic patients. Clin Microbiol Infect 11 (Suppl 5): 17-23, 2005.

9. Kern WV: Risk assessment and treatment of low-risk patients with febrile neutropenia. Clin Infect Dis 42: 533-540, 2006.

10. Klastersky J, Paesmans M, Georgala A, et al: Outpatient oral antibiotics for febrile neutropenic cancer patients using a score predictive for complications. J Clin Oncol 24: 4129-4134, 2006.

11. Bow EJ, Rotstein C, Noskin GA, Laverdiere M, Schwarer AP, Segal BH, Seymour JF, Szer J and Sanche S: A randomized, open label, multicenter comparative study of the efficacy and safety of piperacillin-tazobactam and cefepime for the empirical treatment of febrile neutropenic episodes in patients with hematologic malignancies. Clin Infect Dis 43: 447-459, 2006.

12. Freifeld AG, Bow EJ, Sepkowitz KA, Boeckh MJ, Ito JI, Mullen CA, Raad II, Rolston KV, Young JA and Wingard JR; Infectious Diseases Society of America: Clinical practice guideline for the use of antimicrobial agents in neutropenic patients with cancer: 2010 update by the Infectious Diseases Society of America. Clin Infect Dis 52: e56-e93, 2011.

13. Paesmans M: Risk factors assessment in febrile neutropenia. Int J Antimicrob Agents 16: 107-111, 2000.

14. Kamana M, Escalante C, Mullen CA, Frisbee-Hume S and Rolston KV: Bacterial infections in low-risk, febrile neutropenic patients. Cancer 104: 422-426, 2005.

15. Günalp M, Koyunoğlu M, Gürler S, Koca A, Yesilkaya I, Öner E, Akkaş M, Metin Aksu N, Demirkan A, Polat O and Elhan AH: Independent factors for prediction of poor outcomes in patients with febrile neutropenia. Med Sci Monit 20: 1826-1832, 2014.

16. Hughes WT, Armostrong D, Bodey GP, Bow EJ, Brown AE, Calandra T, Feld R, Pizzo PA, Rolston KV, Shenep JL and Young LS: 2002 Guidelines for the use of antimicrobial agents in neutropenic patients with cancer. Clin Infect Dis 34: 730-751, 2002. 
17. Zinner SH: Changing epidemiology of infections in patients with neutropenia and cancer: Emphasis on gram-positive and resistant bacteria. Clin Infect Dis 29: 490-494, 1999.

18. Wisplinghoff H, Seifert H, Wenzel RP and Edmond MB: Current trends in the epidemiology of nosocomial bloodstream infections in patients with hematological malignancies and solid neoplasms in hospitals in the United States. Clin Infect Dis 36: 1103-1110, 2003.
19. Morris PG, Hassan T, McNamara M, Hassan A, Wiig R, Grogan L, Breathnach OS, Smyth E and Humphreys H: Emergence of MRSA in positive blood cultures from patients with febrile neutropenia - a cause for concern. Support Care Cancer 16: 1085-1088, 2008. 